\title{
Green function of the double-fractional Fokker-Planck equation: Path integral and stochastic differential equations
}

\author{
H. Kleinert* \\ Institut für Theoretische Physik, Freie Universität Berlin, 14195 Berlin, Germany \\ and ICRANeT, Piazzale della Repubblica, 10, 65122 Pescara, Italy \\ V. Zatloukal \\ and Max Planck Institute for the History of Science, Boltzmannstrasse 22, 14195 Berlin, Germany
}

Faculty of Nuclear Sciences and Physical Engineering, Czech Technical University in Prague, Břehová 7, 115 19 Praha 1, Czech Republic

(Received 11 July 2013; published 6 November 2013)

\begin{abstract}
The statistics of rare events, the so-called black-swan events, is governed by non-Gaussian distributions with heavy power-like tails. We calculate the Green functions of the associated Fokker-Planck equations and solve the related stochastic differential equations. We also discuss the subject in the framework of path integration.
\end{abstract}

DOI: 10.1103/PhysRevE.88.052106

PACS number(s): 05.40.Fb, 05.10.Gg, 11.15.Me

\section{INTRODUCTION}

Gaussian random walks prove to be a natural and rather universal starting point for many stochastic processes. In fact, the famous central-limit theorem shows that many independent random movements of finite variance $\sigma^{2}=\left\langle x^{2}\right\rangle$ always pile up to display a Gaussian distribution [1]. In particular, Gaussian random walks constitute the basis of the most important tool in the theory of financial markets, the Black-Scholes option price theory [2], by which a portfolio of assets hopefully remains growing steadily through hedging [3].

However, since the last stock market crash and the still ongoing financial crisis, it has become clear that distributions which realistically describe the behavior of financial markets belong to a more general universality class, the so-called Lévy stable distribution [4-6]. They result from a sum of random movements of infinite variance [7] and account for the fact that rare events, the so-called black-swan events [8], which initiate crashes, are much more frequent than in Gaussian distributions. These are events in the so-called Lévy tails $\propto 1 /|x|^{1+\lambda}$ of the distributions, whose description is based on a generalized Hamiltonian [9]:

$$
H(p)=\operatorname{const}\left(p^{2}\right)^{\lambda / 2} .
$$

Such tail events are present in many physical situations, e.g., in velocity distributions of many-body systems with long-range forces [10], in the self-similar distribution of matter in the universe [11-13], and in the distributions of wind gusts [14] and earthquakes [15], with often catastrophic consequences.

Distributions with Lévy tails are a consequence of rather general maximal entropy assumptions [16]. In the limit $\lambda \rightarrow 2$, the Lévy distributions reduce to Gaussian distributions.

The simplest Lévy-type random walk is described by the stochastic differential equation of the Langevin type,

$$
\frac{d}{d s} x(s) \equiv \dot{x}(s)=\eta(s),
$$

where $\eta(s)$ is a noise variable as a function of a pseudotime $s$ with zero expectation value and a probability distribution

\footnotetext{
*h.k@fu-berlin.de
}

characterized by a parameter $\lambda[17]$ :

$$
P[\eta] \equiv e^{-\int d s \tilde{H}(\eta)}=\int \mathcal{D} p \exp \left\{\int d s\left[i p \eta-\left(p^{2}\right)^{\lambda / 2}\right]\right\}
$$

Using this, we may solve the stochastic differential equation (2) in which the noise $\eta(s)$ has nonzero correlation functions for even $n=2,4,6, \ldots$ :

$$
\left\langle\eta\left(s_{1}\right) \cdots \eta\left(s_{n}\right)\right\rangle \equiv \int \mathcal{D} \eta \eta\left(s_{1}\right) \cdots \eta\left(s_{n}\right) P[\eta] .
$$

For $\lambda=2$, the distribution is Gaussian, and $\eta(s)$ is a standard white noise variable. If we solve (2) in $D$ dimensions with an initial condition $\mathbf{x}(0)=\mathbf{0}$, the variable $\mathbf{x}(s)$ has a distribution

$$
P_{\mathrm{G}}(\mathbf{x}, s)=(4 \pi s)^{-D / 2} e^{-\mathbf{x}^{2} / 4 s} .
$$

This distribution is the Green function of the Fokker-Planck equation,

$$
\left(\partial_{s}+\hat{\mathbf{p}}^{2}\right) P_{G}(\mathbf{x}, s)=\delta(s) \delta^{(D)}(\mathbf{x}),
$$

where $\hat{\mathbf{p}} \equiv i \partial_{\mathbf{x}} \equiv i \nabla$. For $\lambda \neq 2$, the distribution is nonGaussian, and it solves the fractional Fokker-Planck equation,

$$
\left[\partial_{s}+\left(\hat{\mathbf{p}}^{2}\right)^{\lambda / 2}\right] P(\mathbf{x}, s)=\delta(s) \delta^{(D)}(\mathbf{x}) .
$$

A solution of this equation that evolves from the $\delta$ function is

$$
P(\mathbf{x}, s)=e^{-s\left(\hat{\mathbf{p}}^{2}\right)^{\lambda / 2}} \delta^{(D)}(\mathbf{x}),
$$

and for $s=1$ it coincides with the noise probability,

$$
\left.P(\mathbf{x}, 1)\right|_{\mathbf{x}=\eta}=P(\boldsymbol{\eta})=\int \frac{d^{D} p}{(2 \pi)^{D}} e^{i \mathbf{p} \cdot \boldsymbol{\eta}-\left(\mathbf{p}^{2}\right)^{\lambda / 2}} .
$$

Applications of the fractional Fokker-Planck equation are numerous in non-Brownian diffusion processes. These are observed in chaotic systems and in the fluid dynamics of rheology and biology. See [18,19] for an overview. The mathematics of Eq. (7) with a variable diffusion coefficient is in [20].

The fractional Fokker-Planck equation (7) can be generalized further to the double-fractional Fokker-Planck equation,

$$
\left[\hat{p}_{4}^{1-\gamma}+D_{\lambda}\left(\hat{\mathbf{p}}^{2}\right)^{\lambda / 2}\right] P(\mathbf{x}, t)=\delta(t) \delta^{(D)}(\mathbf{x}),
$$


where $\hat{p}_{4} \equiv \partial_{t}, \hat{\mathbf{p}} \equiv i \partial_{\mathbf{x}} \equiv i \nabla$, and a parameter has been allowed for that is the analog of the diffusion constant $D$ in the ordinary diffusion process [21].

We should explain the physical origin of the fractional powers in the space and time derivatives of the above equation. Such powers occur naturally in many-particle systems if the interaction strength or the range becomes very large. As long as the interaction strength is small and the range is short, such systems are described by a second-quantized field theory with a free-particle action,

$$
\mathcal{A}_{0}=\int d t d^{3} x \psi^{\dagger}(\mathbf{x}, t)\left[i \partial_{t}+\hbar^{2} \nabla^{2} / 2 m-V(\mathbf{x})\right] \psi(\mathbf{x}, t),
$$

and an interaction of the type

$$
\mathcal{A}_{\text {int }}=\frac{g}{4 !} \int d t d^{3} x\left(\psi^{\dagger} \psi\right)^{2}
$$

The partition function can be calculated from the functional integral

$$
Z=\oint \mathcal{D} \psi \mathcal{D} \psi^{\dagger} e^{i\left(\mathcal{A}_{0}+\mathcal{A}_{\text {int }}\right) / \hbar}
$$

A perturbation expansion leads to an effective action in the form of a power series of $g \Psi^{\dagger} \Psi$, where $\Psi=\langle\psi\rangle$ are the expectation values of the field. This series is divergent and must be resummed. For large interaction strength $g$, this produces anomalous power behaviors in the field strength as well as in the momenta $[22,23]$. The free-field part of the effective action leads to a field equation of the fractional Fokker-Planck or Schrödinger type, in which momentum and energy appear with powers different from $\lambda=2$ and $\gamma=0$, respectively.

In addition, equations of the type of (10) are known to govern various different phenomena. In chaotic systems, for example, they describe anomalous diffusion processes with memory (time nonlocality) [24,25]. In fact, the fractional time derivatives also arise as the infinitesimal generators of coarse grained time evolutions [26], or they can be derived from a random walk model when the mean waiting time of the walker diverges [27].

It is the purpose of this paper to calculate the Green functions of general fractional Fokker-Planck equation (10) and to specify the path integrals solved by them $[28,29]$.

\section{DOUBLE-FRACTIONAL FOKKER-PLANCK EQUATION}

A convenient definition of the fractional derivatives uses the same formula as in the dimensional continuation of Feynman diagrams [30,31],

$$
\left(\hat{\mathbf{p}}^{2}\right)^{\lambda / 2}=\Gamma[-\lambda / 2]^{-1} \int d \sigma \sigma^{-\lambda / 2-1} e^{\sigma \hat{\mathbf{p}}^{2}} .
$$

The solution of (10) can be written formally as

$$
P(\mathbf{x}, t)=\left[\left(\hat{p}_{4}+\epsilon\right)^{1-\gamma}+D_{\lambda}\left(\hat{\mathbf{p}}^{2}\right)^{\lambda / 2}\right]^{-1} \delta(t) \delta^{(D)}(\mathbf{x}),
$$

where infinitesimal $\epsilon>0$ ensures the forward-in-time nature of the Green function, and its explicit appearance will be suppressed from now on. Using the representation $\delta(t)=$ $\int_{-\infty}^{+\infty} \frac{d E}{2 \pi} e^{-i E t}$, we arrive at

$$
P(\mathbf{x}, t)=\int \frac{d E}{2 \pi} \frac{e^{-i E t}}{(-i E)^{1-\gamma}+D_{\lambda}\left(\hat{\mathbf{p}}^{2}\right)^{\lambda / 2}} \delta^{(D)}(\mathbf{x}) .
$$

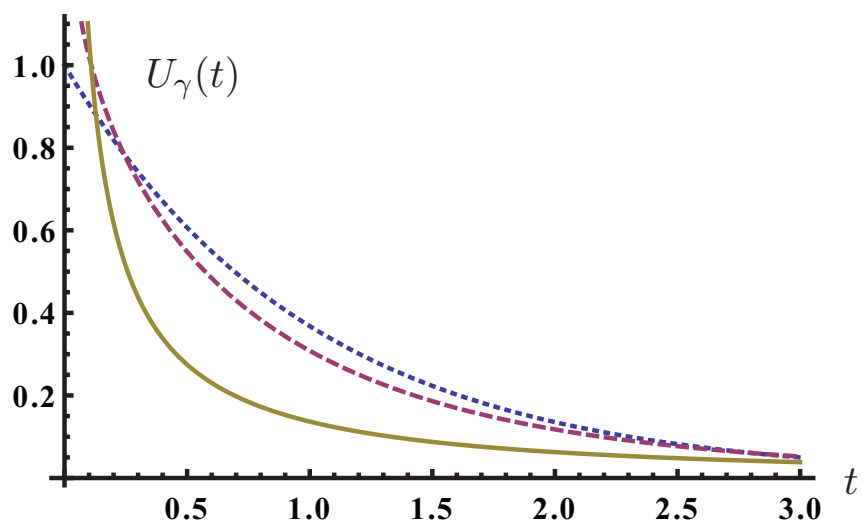

FIG. 1. (Color online) Function $U_{\gamma}(t)$ for $\hat{H}=1$ and various values of $\gamma$. Dotted blue curve: $\gamma=0$, standard exponential function; dashed red curve: $\gamma=0.1$; solid yellow curve: $\gamma=0.5$.

Now we expand the fraction into a geometric series and integrate term by term using the formula [32]

$$
\int_{-\infty}^{+\infty} \frac{d E}{2 \pi} \frac{e^{-i E t}}{(-i E+\epsilon)^{(1-\gamma)(n+1)}}=\frac{\theta(t) t^{n(1-\gamma)-\gamma}}{\Gamma[(1-\gamma)(n+1)]},
$$

where $\theta(t)$ is the Heaviside step function. The result can be cast as

$$
P(\mathbf{x}, t)=\theta(t) t^{-\gamma} E_{1-\gamma, 1-\gamma}\left[-t^{1-\gamma} D_{\lambda}\left(\hat{\mathbf{p}}^{2}\right)^{\lambda / 2}\right] \delta^{(D)}(\mathbf{x}),
$$

where $E_{\alpha, \beta}(z)=\sum_{n=0}^{\infty} \frac{z^{n}}{\Gamma(\alpha n+\beta)}$ is the Mittag-Leffler function $[33,34]$. This can be interpreted by writing

$$
P(\mathbf{x}, t)=\left\langle\mathbf{x}\left|\hat{U}_{\gamma}(t)\right| \mathbf{0}\right\rangle,
$$

with the $\gamma$-deformed evolution $\hat{U}_{\gamma}$ defined by

$$
\hat{U}_{\gamma}(t)=\theta(t) t^{-\gamma} E_{1-\gamma, 1-\gamma}\left(-t^{1-\gamma} \hat{H}\right),
$$

with $\hat{H} \equiv D_{\lambda}\left(\hat{\mathbf{p}}^{2}\right)^{\lambda / 2}$ [35] (see Fig. 1). The occurrence of the Mittag-Leffler function in solutions of the time-fractional Fokker-Planck equation has been noted previously, for example, in the review article by Srokowski [20].

For $\gamma=0$, Eq. (10) reduces to a single (space) fractional Fokker-Planck equation,

$$
\left[\hat{p}_{4}+D_{\lambda}\left(\hat{\mathbf{p}}^{2}\right)^{\lambda / 2}\right] P(\mathbf{x}, t)=\delta^{(D)}(\mathbf{x}) \delta(t),
$$

the Mittag-Leffler function reduces to $E_{1,1}(z)=\exp (z)$, and the evolution operator recovers its standard form $\hat{U}_{0}(t)=$ $\theta(t) \exp (-t \hat{H})$. The solution, which we shall denote by $P_{X}(\mathbf{x}, t)$ for a more specific reference, is the multivariate Lévy stable distribution [36]:

$$
P_{X}(\mathbf{x}, t)=\int \frac{d^{D} p}{(2 \pi)^{D}} e^{-t D_{\lambda}\left(\mathbf{p}^{2}\right)^{\lambda / 2}} e^{-i \mathbf{p} \cdot \mathbf{x}} .
$$

For $\lambda=2$, it reduces to the standard quantum mechanical Gaussian expression (5). For $\lambda=1$, the result is

$$
P_{X}(\mathbf{x}, t)=\frac{\left[\Gamma(D / 2+1 / 2) / \pi^{(D+1) / 2}\right] D_{\lambda} t}{\left[\left(D_{\lambda} t\right)^{2}+|\mathbf{x}|^{2}\right]^{D / 2+1 / 2}},
$$

which is the Cauchy-Lorentz distribution function. In Fig. 2, we plot $P_{X}$ in $D=1$ dimension for $\lambda=1,1.5,2$.

In Appendix B we provide various useful representations of $P_{X}(\mathbf{x}, t)$. It is worth mentioning that this probability can be 


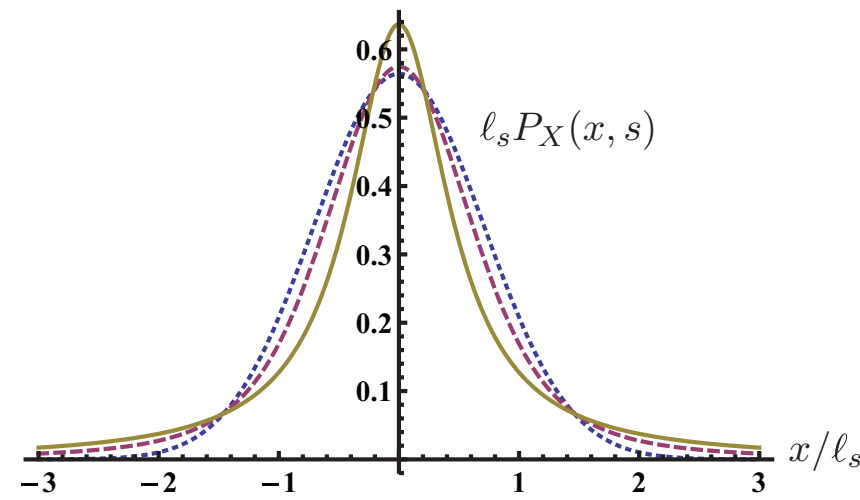

FIG. 2. (Color online) Dotted blue curve: $\lambda=2$, standard Gaussian distribution; dashed red curve: $\lambda=1.5$; solid yellow curve: $\lambda=$ 1 , Cauchy-Lorenz distribution. The length scale is $\ell_{s}=2\left(D_{\lambda} s\right)^{1 / \lambda}$.

written as a superposition of Gaussian distributions $P_{\mathrm{G}}(\sigma, \mathbf{x})=$ $(4 \pi \sigma)^{-D / 2} e^{-\mathbf{x}^{2} / 4 \sigma}$ to be specified in Eq. (B1).

\section{A. Smeared-time representation and relation between physical time $t$ and pseudotime $s$}

If we use in (16) Schwinger's formula $1 / A=\int_{0}^{\infty} d s e^{-s A}$, we can express $P(\mathbf{x}, t)$ as an integral:

$$
P(\mathbf{x}, t)=\int_{0}^{\infty} d s P_{X}(\mathbf{x}, s) P_{T}(t, s),
$$

where $P_{X}$ solves the space-fractional diffusion equation (21), with $t \equiv s$, and $P_{T}$ solves the time-fractional equation

$$
\left[\partial_{s}+\hat{p}_{4}^{1-\gamma}\right] P_{T}(t, s)=\delta(t) \delta(s)
$$

which encodes the relation between the pseudotime $s$ and the physical time $t$. The factorized ansatz (24) has been used previously in [37] to solve the time-fractional Fokker-Planck equation.

For $\gamma=0, P_{T}(t, s)=\delta(t-s)$, and (24) reduces to $P(\mathbf{x}, t)=P_{X}(\mathbf{x}, t)$.

For $\gamma>0$, we obtain an asymmetric Lévy stable distribution [38]

$$
P_{T}(t, s)=\int_{-\infty}^{\infty} \frac{d E}{2 \pi} e^{-s(-i E)^{1-\gamma}} e^{-i E t} .
$$

An important feature is that $P_{T}(t, s)$ vanishes for $t<0$. This can be seen by placing the branch cut of a multivalued function $z^{1-\gamma}$ along the negative real axis and calculating (26) as a complex integral with a contour that follows the real axis and closes in the upper half plane. See Fig. 3(a), where $P_{T}$ is plotted as a function of $t$ for the case $\gamma=0.03$ and various values of $s$.

It is illustrative to view formula (24) as a smearing of the distribution $P_{X}(\mathbf{x}, s)$ around the time position $t$, defined by the probability density function $P_{T}(t, s)$. For this purpose we plot in Fig. 3(b) $P_{T}(t, s)$ as a function of $s$, with parameter $t$ describing the position of the peak in the probability distribution.

The two plots in Fig. 3 are related through the formula

$$
P_{T}(t, s)=(C / t) P_{T}\left(C, C^{1-\gamma} t^{\gamma-1} s\right),
$$

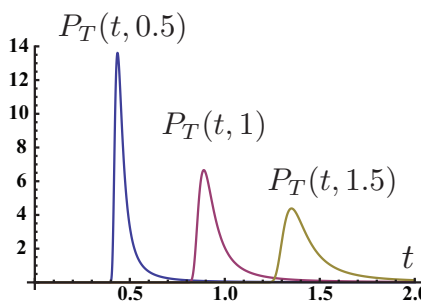

(a)

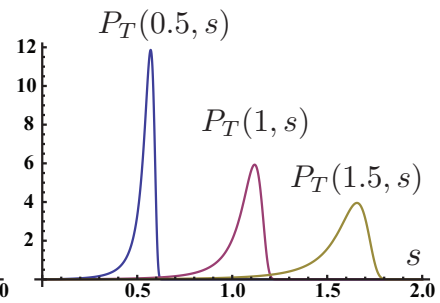

(b)
FIG. 3. (Color online) (a) $P_{T}(t, s)$ as a distribution of $t$ with increasing values of the pseudotime $s=0.5,1,1.5$. (b) $P_{T}(t, s)$ as a distribution of $s$ with increasing values of the real time $t=0.5,1,1.5$. In both cases $\gamma=0.03$.

which can be deduced from (26) by a simple change of the integration variable $E \rightarrow(C / t) E$. Here $C$ is an arbitrary constant. The function $P_{T}(t, s)$ as a function of two variables is shown in Fig. 4.

When $\gamma=0, P_{T}(t, s)=\delta(t-s)$ is concentrated at point $t$; that is, there is no smearing. For increasing $\gamma$ the peak around $t$ broadens, which can be accounted for by derivatives of the $\delta$ function. The action of $P_{T}$ on a test function $f(s)$ is

$\int_{0}^{\infty} d s P_{T}(t, s) f(s)=\sum_{n=0}^{\infty} \frac{f^{(n)}(t)}{n !} \int_{0}^{\infty} d s P_{T}(t, s)(s-t)^{n}$.

We represent $f^{(n)}(t)=(-1)^{n} \int d \tau \delta^{(n)}(\tau-t) f(\tau)$ and calculate

$$
\int_{0}^{\infty} d s P_{T}(t, s) s^{k}=\int \frac{d E}{2 \pi} \frac{e^{-i E t} k !}{(-i E)^{(1-\gamma)(k+1)}}=\frac{k ! \theta(t) t^{(1-\gamma) k-\gamma}}{\Gamma[(1-\gamma)(k+1)]}
$$

to find that

$$
P_{T}(t, s)=\sum_{n=0}^{\infty} \frac{t^{n}}{n !} c_{n}(t) \delta^{(n)}(s-t)
$$

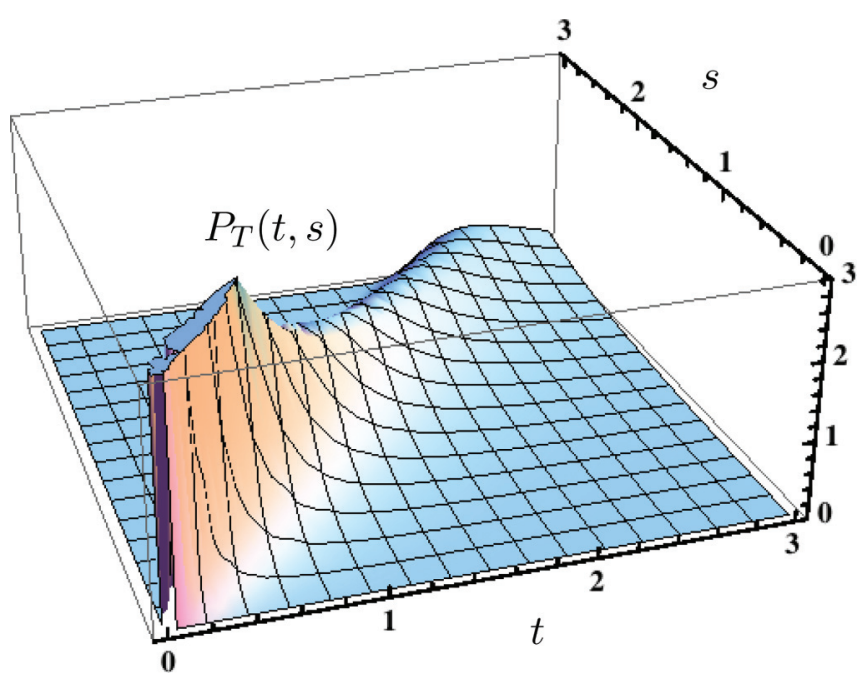

FIG. 4. (Color online) $P_{T}(t, s)$ as a function of both $t$ and $s$. Here $\gamma=0.1$. 


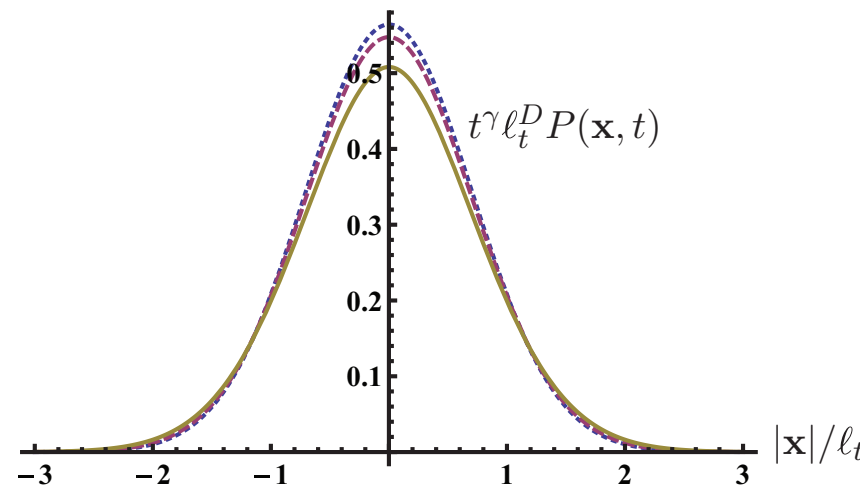

FIG. 5. (Color online) In all cases $\lambda=2$. Dotted blue curve: $\gamma=$ 0 , standard Gaussian distribution; dashed red curve: $\gamma=0.03$; solid yellow curve: $\gamma=0.1$.

where

$$
c_{n}(t)=\sum_{k=0}^{n}\left(\begin{array}{l}
n \\
k
\end{array}\right)(-1)^{k} \frac{k ! \theta(t) t^{-\gamma(k+1)}}{\Gamma[(1-\gamma)(k+1)]} .
$$

In view of these relations, Eq, (24) translates into

$$
P(\mathbf{x}, t)=\sum_{n=0}^{\infty} \frac{(-t)^{n}}{n !} c_{n}(t) \partial_{t}^{n} P_{X}(\mathbf{x}, t) .
$$

One can easily verify that for $\gamma=0, c_{n}=\delta_{n 0}$, and $P(\mathbf{x}, t)=$ $P_{X}(\mathbf{x}, t)$.

\section{B. Fox $\boldsymbol{H}$ function representation of Green function}

A solution of the double-fractional equation (10) has been obtained previously in terms of the Fox $H$ function [39]. We derive the same result starting from formula (24), where we consider the representation [Eq. (B4)] of $P_{X}(\mathbf{x}, s)$. Integration over the pseudotime $s$ can be performed, followed by the $E$ integration, which yields

$$
P(\mathbf{x}, t)=\frac{t^{-\gamma}}{\pi^{D / 2}|\mathbf{x}|^{D}} H_{2,3}^{2,1}\left(\left.\left[\frac{|\mathbf{x}|}{\ell_{t}}\right]^{\lambda}\right|_{(1,1),(D / 2, \lambda / 2) ;(1, \lambda / 2)} ^{(1,1) ;(1-\gamma, 1-\gamma)}\right) .
$$

Here $\ell_{t} \equiv 2\left(D_{\lambda} t^{1-\gamma}\right)^{1 / \lambda}$ is a $t$-dependent length scale, and $H_{2,3}^{2,1}$ is the Fox $H$ function [40,41], defined by the contour integral

$$
\frac{P(\mathbf{x}, t)|\mathbf{x}|^{D}}{t^{-\gamma} \pi^{-D / 2}}=\int_{\mathcal{C}} \frac{d z}{2 \pi i} \frac{\Gamma(1+z) \Gamma\left(\frac{D}{2}+\frac{\lambda}{2} z\right) \Gamma(-z)}{\Gamma\left(-\frac{\lambda}{2} z\right) \Gamma(1-\gamma+(1-\gamma) z)}\left[\frac{|\mathbf{x}|^{\lambda}}{\ell_{t}^{\lambda}}\right]^{-z},
$$

where the contour $\mathcal{C}$ runs from $-i \infty$ to $+i \infty$. In Fig. 5 we show how values of $\gamma>0$ modify the Gaussian distribution (for which $\lambda=2, \gamma=0$ ).

The large- $|\mathbf{x}|$ asymptotics of (33) is governed by the pole of the integrand at $z=1$ :

$$
t^{\gamma}|\mathbf{x}|^{D} P(\mathbf{x}, t) \stackrel{|\mathbf{x}| \rightarrow \infty}{\approx} \frac{\ell_{t}^{\lambda}}{|\mathbf{x}|^{\lambda}} \frac{-\Gamma\left(\frac{D+\lambda}{2}\right)}{\pi^{D / 2} \Gamma(2-2 \gamma) \Gamma\left(-\frac{\lambda}{2}\right)} .
$$

Analysis of the small- $|\mathbf{x}|$ behavior is more subtle due to a richer pole structure of the integrand in (34) (see [42]). If we

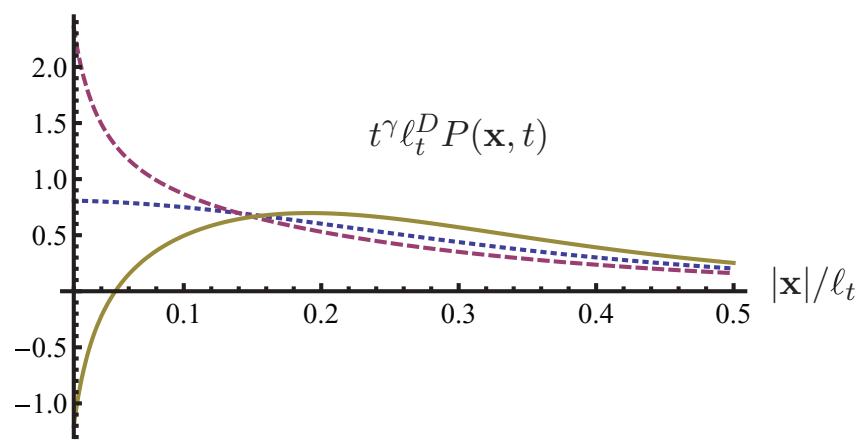

FIG. 6. (Color online) Dotted blue curve: $\gamma=0, \lambda=1$, CauchyLorentz distribution; dashed red curve: $\gamma=0.1, \lambda=1$; solid yellow curve: $\gamma=-0.1, \lambda=1$, assumes negative values. Here $D=3$.

assume only simple poles, we can extract the leading behavior

$$
t^{\gamma} P(\mathbf{x}, t) \stackrel{|\mathbf{x}| \rightarrow 0}{\approx}\left\{\begin{array}{ll}
A(t)+B(t)|\mathbf{x}|^{2 \lambda-D}, & 2 \lambda-D<2 \\
A(t)+\mathcal{O}\left[|\mathbf{x}|^{2}\right](t), & 2 \lambda-D>2
\end{array},\right.
$$

with

$$
\begin{gathered}
A(t)=\frac{\pi^{1-D / 2} \ell_{t}^{-D} 2 / \lambda}{\sin \left(\pi \frac{D}{\lambda}\right) \Gamma\left(\frac{D}{2}\right) \Gamma\left[\frac{(1-\gamma)(\lambda-D)}{\lambda}\right]}, \\
B(t)=-\frac{\pi^{-D / 2} \Gamma\left(\frac{D}{2}-\lambda\right)}{\Gamma(\lambda) \Gamma(\gamma-1) \ell_{t}^{2 \lambda}} .
\end{gathered}
$$

In particular, for $2 \lambda<D$ the value of $P(\mathbf{x}, t)$ tends to either $+\infty$ or $-\infty$ as $|\mathbf{x}| \rightarrow 0$. See Fig. 6 .

\section{PATH-INTEGRAL FORMULATION}

We note that the probability (15) may be calculated from the doubly fractional canonical path integral over fluctuating orbits $t(s), \mathbf{x}(s), p_{4}(s), \mathbf{p}(s)$ viewed as functions of some pseudotime $s[43]:$

$$
\left\{\mathbf{x}_{b} t_{b} s_{b} \mid \mathbf{x}_{a} t_{s} s_{a}\right\}=\int \mathcal{D} \mathbf{x} \mathcal{D} t \mathcal{D} \mathbf{p} \mathcal{D} p_{4} e^{\mathcal{A}},
$$

with $\mathcal{A}$ being the Euclidean action of the paths $t(s), \mathbf{x}(s)$ :

$$
\mathcal{A}=\int d s\left[i\left(\mathbf{p} \mathbf{x}^{\prime}-i p_{4} t^{\prime}\right)-\mathcal{H}\left(\mathbf{p}, p_{4}\right)\right] .
$$

Here $t^{\prime}(s) \equiv d t(s) / d s, \quad \mathbf{x}^{\prime}(s) \equiv d \mathbf{x}(s) / d s$, and $\mathcal{H}\left(\mathbf{p}, p_{4}\right)=$ $p_{4}^{1-\gamma}+D_{\lambda}\left(\hat{\mathbf{p}}^{2}\right)^{\lambda / 2}$. At each $s$, the integrals over the components of $\mathbf{p}(s)$ run from $-\infty$ to $\infty$, whereas those over $p_{4}(s)$ run from $-i \infty$ to $i \infty$. To obtain the distribution $P(\mathbf{x}, t)$, we finally form the integral

$$
P(\mathbf{x}, t)=\int_{0}^{\infty} d s\left\{\begin{array}{lllll}
\mathbf{x} t & \mid \mathbf{0} & 0 & 0
\end{array}\right\} .
$$

This is analogous to prescription (24), which links solutions of the space- and time-fractional diffusion equations (21) and (25).

If $\gamma=0$, the path integral over $p_{4}(s)$ yields the functional $\delta\left[t^{\prime}(s)-1\right]$, which ensures that $d t$ and $d s$ increments are equal. This brings (39) to the canonical path integral

$$
\left(\mathbf{x}_{b} t_{b} \mid \mathbf{x}_{a} t_{a}\right)=\int \mathcal{D} \mathbf{x} \mathcal{D} \mathbf{p} e^{\mathcal{A}^{\prime}}
$$


with

$$
\mathcal{A}^{\prime}=\int d \tau\left[i \mathbf{p} \cdot \dot{\mathbf{x}}-D_{\lambda}\left(\hat{\mathbf{p}}^{2}\right)^{\lambda / 2}\right]
$$

Now $P(\mathbf{x}, t)=(\mathbf{x} t \mid \mathbf{0} 0)$ satisfies the ordinary fractional FokkerPlanck equation

$$
\left[\hat{p}_{4}+D_{\lambda}\left(\hat{\mathbf{p}}^{2}\right)^{\lambda / 2}\right] P(\mathbf{x}, t)=\delta(t) \delta^{(D)}(\mathbf{x}),
$$

which has been discussed at length in recent literature [44].

\section{LANGEVIN EQUATIONS AND COMPUTER SIMULATIONS}

In the past, many nontrivial Schrödinger equations (for instance, that of the $1 / r$ potential) have been solved with pathintegral methods by reformulating them on the pseudotime axis $s$, which is related to the time $t$ via a space-dependent differential equation $t^{\prime}(s)=f(x(t))$. This method was invented by Duru and Kleinert [45] to solve the path integral of the hydrogen atom and has recently been applied successfully to various Fokker-Planck equations [46,47]. The stochastic differential equation (47), which connects pseudotime $s$ and the physical time $t$, may be seen as a stochastic version of the Duru-Kleinert transformation that promises to be a useful tool to study non-Markovian systems.

Certainly, the solutions of Eq. (44) can also be obtained from the stochastic differential equation

$$
\dot{\mathbf{x}}=\eta,
$$

whose noise is distributed with a fractional probability

$$
P[\boldsymbol{\eta}]=\int \mathcal{D}^{D} p e^{\int d t\left[i \mathbf{p} \cdot \boldsymbol{\eta}-D_{\lambda}\left(\mathbf{p}^{2}\right)^{\lambda / 2}\right]} .
$$

Simulating this stochastic differential equation on a computer, we confirm the analytic form (22) of $P_{X}(\mathbf{x}, s)=P(\mathbf{x}, t)$ for $\gamma=0$. See Fig. 7(a).

Analogously, the solution of Eq. (25) can also be obtained from the stochastic differential equation

$$
t^{\prime}(s)=\eta_{T}(s),
$$

with noise distribution

$$
P\left[\eta_{T}\right]=\int \mathcal{D} p_{4} e^{\int d s\left[p_{4} \eta_{T}-\left(p_{4}\right)^{1-\gamma}\right]},
$$

and compared with the result (26) for $P_{T}(t, s)$. See Fig. 7(b).

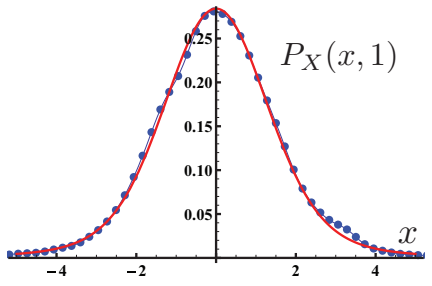

(a)

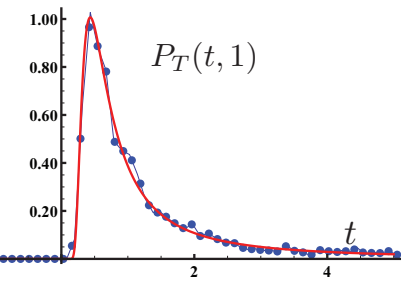

(b)
FIG. 7. (Color online) Comparison of analytic (solid red curve) and numerical (blue circles) results for (a) the distribution function $P_{X}(x, s=1)$ in $D=1$ dimension and (b) $P_{T}(t, s=1)$ for $\gamma=0.3$. In each case an average has been taken over 5000 representative trajectories of stochastic differential equations (45) and (47), with 10 time steps $\Delta s=0.1$.

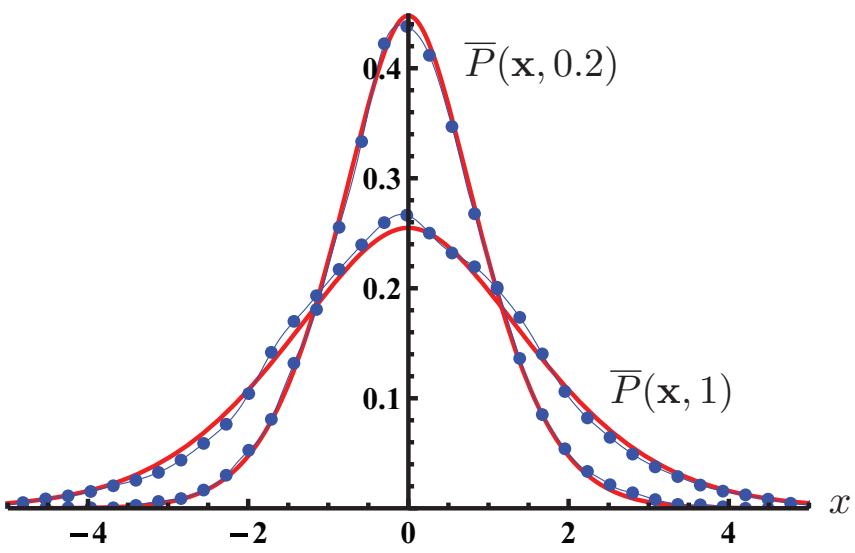

FIG. 8. (Color online) Comparison of computer simulation and the renormalized exact solution $\bar{P}(\mathbf{x}, t)$ for $t=0.2,1$.

The solution of the double-fractional Fokker-Planck equation (10) can be obtained, in view of relation (41) [or (24)], by simulating (45) for $t \equiv s$ and (47) and letting the final value of the pseudotime $s$ be random. This yields a probability distribution $P(\mathbf{x}, t)$. In Fig. 8 we compare the results of a computer simulation with the analytic form (34) by plotting $P(\mathbf{x}, t)$ as a function of $\mathbf{x}$ for various values of time $t$. Since the distribution $P(\mathbf{x}, t)$ itself is not normalized, but rather

$$
\int d^{D} x P(\mathbf{x}, t)=\int_{0}^{\infty} d s P_{T}(t, s)=\frac{\theta(t) t^{-\gamma}}{\Gamma(1-\gamma)},
$$

we define a renormalized version $\bar{P}(\mathbf{x}, t)=P(\mathbf{x}, t)$ / $\int d^{D} x P(\mathbf{x}, t)$.

\section{SUMMARY}

Summarizing, we have seen that a many-body system with strong couplings between the constituents satisfies a more general form of the Schrödinger equation, in which the momentum and the energy appear with a power different from $\lambda=2$ and $\gamma=0$, respectively. We have calculated the associated Green functions and discussed their properties and their representations. We have pointed out that these Green functions can be written as path integrals over fluctuating time and space orbits that are functions of some pseudotime $s$. This is a Markovian object but is non-Markovian in the physical time $t$. The non-Markovian character is caused by the fact that function $t(s)$ follows a stochastic differential equation of the Langevin type.

The particle distributions can also be obtained by solving a Langevin type of equation in which the noise has correlation functions whose probability distribution is specified by an equation like (46).

The Green functions whose theory was presented here will play an important role in the development of an interacting theory of fields whose world lines contain non-Gaussian random walks displaying extremely large deviations from their averages.

\section{ACKNOWLEDGMENTS}

We are grateful to P. Jizba and A. Pelster for useful comments. One of the authors (V.Z.) is grateful for the financial 
support from the Deutsche Forschungsgemeinschaft, Grant KL 256/54-1, and the Czech Science Foundation (GAČR), Grant No. P402/12/J077.

\section{APPENDIX A}

Fractional differential operators that enter the general fractional Fokker-Planck equation (7) are defined through formula (14). Using $e^{-\sigma \hat{\mathbf{p}}^{2}} \delta(\mathbf{x})=(4 \pi \sigma)^{-D / 2} e^{-\mathbf{x}^{2} /(4 \sigma)}$ and $e^{-\sigma \hat{p}_{4}} \delta(t)=$ $\delta(t-\sigma)$, we derive the following relations:

$$
\begin{gathered}
|\mathbf{x}|^{\lambda}=\frac{\pi^{D / 2} \Gamma\left(\frac{\lambda+D}{2}\right)}{2^{-\lambda-D} \Gamma\left(-\frac{\lambda}{2}\right)}\left(\hat{\mathbf{p}}^{2}\right)^{-(\lambda+D) / 2} \delta^{(D)}(\mathbf{x}), \\
\theta(t) t^{\alpha}=\Gamma(\alpha+1)\left(\hat{p}_{4}\right)^{-\alpha-1} \delta(t),
\end{gathered}
$$

which we can substitute into (33) and (34) in order to verify that they satisfy Eq. (10). We first obtain

$$
\begin{aligned}
P(\mathbf{x}, t)= & \int_{\mathcal{C}} \frac{d z}{2 \pi i} \Gamma(1+z) \Gamma(-z) D_{\lambda}^{z}\left(\hat{\mathbf{p}}^{2}\right)^{\lambda z / 2} \\
& \times\left(\hat{p}_{4}\right)^{(\gamma-1)(z+1)} \delta^{(D)}(\mathbf{x}) \delta(t),
\end{aligned}
$$

which can be pole-expanded to yield

$$
\sum_{n=0}^{\infty}\left(-D_{\lambda}\right)^{n}\left(\hat{\mathbf{p}}^{2}\right)^{\lambda n / 2}\left(\hat{p}_{4}\right)^{(\gamma-1)(n+1)} \delta^{(D)}(\mathbf{x}) \delta(t) .
$$

Summing up this geometric series, we arrive at

$$
P(\mathbf{x}, t)=\left[\hat{p}_{4}^{1-\gamma}+D_{\lambda}\left(\hat{\mathbf{p}}^{2}\right)^{\lambda / 2}\right]^{-1} \delta^{(D)}(\mathbf{x}) \delta(t) .
$$

\section{APPENDIX B}

We derive several expressions for the solution $P_{X}(\mathbf{x}, s)$ of (21), starting from representation (22).
On expanding the exponential and representing the powers as $\left(\mathbf{p}^{2}\right)^{\lambda n / 2}=\Gamma[-\lambda n / 2]^{-1} \int_{0}^{\infty} \frac{d \sigma}{\sigma} \sigma^{-\lambda n / 2} e^{-\sigma \mathbf{p}^{2}}$, the momentum integration yields the superposition of Gaussian expression:

$$
P_{X}(\mathbf{x}, s)=\int_{0}^{\infty} \frac{d \sigma}{\sigma} f_{\lambda}(\sigma) P_{G}\left(\mathbf{x}, D_{\lambda}^{2 / \lambda} s^{2 / \lambda} \sigma\right),
$$

with weight

$$
f_{\lambda}(\sigma)=\sum_{n=0}^{\infty} \frac{(-1)^{n} \sigma^{-\lambda n / 2}}{n ! \Gamma(-\lambda n / 2)} .
$$

To prove this, we perform the $\sigma$ integration term by term, using the formula $\int_{0}^{\infty} \frac{d \sigma}{\sigma} \sigma^{-v} e^{-a / \sigma}=\Gamma(v) / a^{v}$, and obtain the large- $|\mathbf{x}|$ expansion,

$$
P_{X}(\mathbf{x}, s)=\frac{1}{\pi^{D / 2}|\mathbf{x}|^{D}} \sum_{n=0}^{\infty} \frac{(-1)^{n} \Gamma\left(\frac{\lambda n+D}{2}\right)}{n ! \Gamma(-\lambda n / 2)}\left[\frac{\ell_{s}^{\lambda}}{|\mathbf{x}|^{\lambda}}\right]^{n},
$$

where $\ell_{s}=2\left(D_{\lambda} s\right)^{1 / \lambda}$. The series can also be viewed as a pole expansion of the contour integral, and hence

$$
P_{X}(\mathbf{x}, s)=\frac{1}{\pi^{D / 2}|\mathbf{x}|^{D}} \int_{\mathcal{C}} \frac{d z}{2 \pi i} \frac{\Gamma\left(\frac{\lambda z+D}{2}\right) \Gamma(-z)}{\Gamma(-\lambda z / 2)}\left[\frac{|\mathbf{x}|^{\lambda}}{\ell_{s}^{\lambda}}\right]^{-z},
$$

with the contour $\mathcal{C}$ running from $-i \infty$ to $+i \infty$. From this, expansion (B3) arises by enclosing the right complex half plane and calculating the residua of the integrand, using $\operatorname{Res}[\Gamma(a z+$ $b),-(n+b) / a]=(-1)^{n} /(n ! a)$. A small- $|\mathbf{x}|$ expansion of (B4) is obtained by closing the integration contour in the left half plane, leading to

$$
P_{X}(\mathbf{x}, s)=\sum_{n=0}^{\infty} \frac{(-1)^{n} 2 / \lambda}{\pi^{D / 2} \ell_{s}^{D}} \frac{\Gamma\left(\frac{2 n+D}{\lambda}\right)}{n ! \Gamma\left(\frac{D}{2}+n\right)}\left[\frac{|\mathbf{x}|^{2}}{\ell_{s}^{2}}\right]^{n} .
$$

The series (B3) and (B5) are convergent or asymptotic or even trivially zero, depending on the parameter $\lambda$.
[1] W. Feller, An Introduction to Probability Theory and Its Applications (Wiley, New York, 1991), Vol. 2; J.-P. Bouchaud and M. Potters, Theory of Financial Risks, from Statistical Physics to Risk Management (Cambridge University Press, Cambridge, 2000). See also Chap. 20 in Ref. [28].

[2] F. Black and M. Scholes, J. Political Econ. 81, 637 (1973).

[3] The theory has been reviewed in many detailed publications; our notation follows [28].

[4] T. Preis, Sci. Cult. 76, 333 (2010).

[5] R. N. Mantegna and H. E. Stanley, Introduction to Econophysics: Correlations and Complexity in Finance (Cambridge University Press, Cambridge, 2000).

[6] B. Podobnik, P. Ch. Ivanov, Y. Lee, A. Chessa, and H. E. Stanley, Europhys. Lett. 50, 711 (2000).

[7] A traveling pedestrian salesman is a Gaussian random walker; as a jet-setter he becomes a Lévy random walker.

[8] See http://en.wikipedia.org/wiki/Black_swan_theory.
[9] The concept of a Hamiltonian in the theory of statistical distributions was introduced in [28]. It has its root in the pathintegral formulation of quantum mechanics and emphasizes the fact that in this formulation particles run along fluctuating world lines in space-time, where they perform random walks with distributions that may be Gaussian or non-Gaussian depending on the form of the Hamiltonian as functions of the momentum $p$. The distributions solve a Fokker-Planck equation of the type of (7), driven by a differential operator that is obtained by replacing the momentum in the Hamiltonian $H(p)$ by the differential operator $-i \partial_{x}$.

[10] C. Nardini, S. Gupta, S. Ruffo, T. Dauxois, and F. Bouchet, J. Stat. Mech. (2012) L01002.

[11] R. E. Angulo, V. Springel, S. D. M. White, A. Jenkins, C. M. Baugh, and C. S. Frenk, Mon. Not. R. Astron. Soc. 426, 2046 (2012).

[12] Du Jiulin, Europhys. Lett. 67, 893 (2004); Phys. Lett. A 329, 262 (2004).

[13] J. Einasto, arXiv:1109.5580. 
[14] F. Boettcher, C. Renner, H. P. Waldl, and J. Peinke, Boundary Layer Meteorol. 108, 163 (2003).

[15] P. Bhattacharyya, A. Chatterjee, and B. K. Chakrabarti, Phys. A 381, 377 (2007).

[16] S. Umarov, C. Tsallis, M. Gell-Mann, and S. Steinberg, J. Math. Phys. 51, 033502 (2010).

[17] Note that function $\tilde{H}(\eta)$ is the functional Fourier transform of the Hamiltonian (1) that drives the Fokker-Planck equation (7). See Eq. (20.153) in Ref. [28].

[18] Lévy Flights and Related Topics in Physics, Lecture Notes in Physics Vol. 450, edited by M. F. Shlesinger, G. M. Zaslavsky, U. Frish (Springer, New York, 1995).

[19] Anomalous Diffusion: From Basics to Applications, Lecture Notes in Physics Vol. 519, edited by R. Kutner, A. Pekalski, K. Sznajd-Weron (Springer, Berlin, 1999).

[20] T. Srokowski, Phys. Rev. E 79, 040104(R) (2009).

[21] Fokker-Planck equations in which only the time derivative has a fractional power have been studied in R. Metzler and J. Klafter, Phys. Rep. 339, 1 (2000).

[22] H. Kleinert, V. Schulte-Frohlinde, and V. Schulte-Frohlinde, Critical Phenomena in $\phi^{4}$-Theory (World Scientific, Singapore, 2001).

[23] H. Kleinert, Europhys. Lett. 100, 10001 (2012); J. Phys. B 46, 175401 (2013).

[24] M. M. Meerschaert and A. Sikorskii, Stochastic Models for Fractional Calculus (de Gruyter, Berlin, 2011).

[25] G. M. Zaslavsky, Hamiltonian Chaos and Fractional Dynamics (Oxford University Press, Oxford, 2005).

[26] Applications of Fractional Calculus in Physics, edited by R. Hilfer (World Scientific, Singapore, 2000).

[27] E. Barkai, R. Metzler, and J. Klafter, Phys. Rev. E 61, 132 (2000).

[28] H. Kleinert, Path Integrals in Quantum Mechanics, Statistics, Polymer Physics, and Financial Markets (World Scientific, Singapore, 2006).

[29] R. P. Feynman, Phys. Rev. 80, 440 (1950); R. P. Feynman and A. R. Hibbs, Quantum Mechanics and Path Integrals (McGrawHill, New York, 1965).

[30] For the so-called Riesz fractional derivative see R. Metzler, E. Barkai, and J. Klafter, Phys. Rev. Lett. 82, 3563 (1999); B. J. West, P. Grigolini, R. Metzler, and T. F. Nonnenmacher, Phys. Rev. E 55, 99 (1997). For the so-called Weyl derivative see R. K. Raina and C. L. Koul, Proc. Am. Math. Soc. 73, 188 (1979).
[31] The relevant functional matrix is $\left\langle\mathbf{x}\left|\left(-\nabla^{2}\right)^{\lambda / 2}\right| \mathbf{x}^{\prime}\right\rangle=$ $\Gamma[-\lambda / 2]^{-1} \int d \sigma \sigma^{-\lambda / 2-1}(4 \pi \sigma)^{-D / 2} e^{R^{2} / 4 \sigma}={ }^{D} c_{\lambda} R^{-\lambda-D}$, where ${ }^{D} c_{\lambda}=2^{\lambda} \Gamma((D+\lambda) / 2) / \pi^{D / 2} \Gamma(-\lambda / 2)$ and $R \equiv\left|\mathbf{x}-\mathbf{x}^{\prime}\right|$. If $\lambda$ is close to an even integer, it needs a small positive shift $\lambda \rightarrow \lambda_{+} \equiv \lambda+\epsilon$, and we can replace $\epsilon R^{\epsilon-1}$ by $\delta(R)=S_{D} R^{D-1} \delta^{(D)}(\mathbf{R}), \quad$ where $\quad S_{D}=2 \pi^{D / 2} / \Gamma(D / 2)$. For $A>0$ we have $\left|\mathbf{x}^{\prime}\right|^{-A}={ }^{D} c_{\lambda_{A}}^{-1}\left\langle\mathbf{x}^{\prime}\left|\left(-\nabla^{2}\right)^{\lambda_{A} / 2}\right| \mathbf{0}\right\rangle$ with $\lambda_{A} \equiv A-D$, so that we find $\int d^{D} x^{\prime}\left\langle\mathbf{x}\left|\left(-\nabla^{2}\right)^{\lambda / 2}\right| \mathbf{x}^{\prime}\right\rangle\left|\mathbf{x}^{\prime}\right|^{-A}=$ ${ }^{D} c_{\lambda_{A}}^{-1}\left\langle\mathbf{x}\left|\left(-\nabla^{2}\right)^{(\lambda+A-D) / 2}\right| \mathbf{0}\right\rangle={ }^{D} c_{\lambda+A-D}{ }^{D} c_{\lambda_{A}}^{-1}|\mathbf{x}|^{-A-\lambda}$.

[32] I. S. Gradshteyn and I. M. Ryzhik, Table of Integrals, Series, and Products, 7th ed. (Elsevier, Amsterdam, 2007), Formula 3.382.7.

[33] A. Erdélyi, W. Magnus, F. Oberhettinger, and F. Tricomi, Higher Transcendental Functions (Robert E. Krieger Publishing Company, Malabar, FL, 1981), Vol. 3, pp. 206-212.

[34] H. J. Haubold, A. M. Mathai, and R. K. Saxena, J. Appl. Math. 2011, 298628 (2011).

[35] More generally, $\hat{H}$ can be a generic time-independent Hamiltonian. In particular, it may contain an additional potential term.

[36] J. P. Nolan, in A Practical Guide to Heavy Tails, edited by R. J. Adler, R. E. Feldman, and M. S. Taqqu (Birkhauser, Boston, 1998), pp. 509-526.

[37] E. Barkai and R. J. Silbey, J. Phys. Chem. B 104, 3866 (2000).

[38] Lévy distributions are implemented in WOLFRAM MATHEMATICA 8 under the command STABLEDISTRIBUTION.

[39] J.-S. Duan, J. Math. Phys. 46, 013504 (2005).

[40] C. Fox, Trans. Am. Math. Soc. 98, 395 (1961).

[41] A. M. Mathai, R. K. Saxena, and H. J. Haubold, The H Function: Theory and Applications (Springer, Berlin, 2010).

[42] A. A. Kilbas and M. Saigo, J. Appl. Math. Stochastic Anal. 12, 191 (1999).

[43] This technique is explained in Chaps. 12 and 19 of Ref. [28]. The pseudotime $s$ resembles the so-called Schwinger proper time used in relativistic physics.

[44] N. Laskin, arXiv:1009.5533; Phys. Lett. A 268, 298 (2000); Phys. Rev. E 62, 3135 (2000); 66, 056108 (2002); Chaos 10, 780 (2000); Commun. Nonlinear Sci. Numer. Simul. 12, 2 (2007).

[45] I. H. Duru and H. Kleinert, Phys. Lett. B 84, 30 (1979); Fortschr. Phys. 30, 401 (1982). See also Chaps. 13 and 14 in Ref. [28].

[46] A. Young and C. DeWitt-Morette, Ann. Phys. (NY) 169, 140 (1986); H. Kleinert and A. Pelster, Phs. Rev. Lett. 78, 565 (1997).

[47] L. Z. J. Liang, D. Lemmens, and J. Tempere, Phys. Rev. E 83, 056112 (2011). 